\title{
Computed tomography simulator conversion curve dependence on scan parameters and phantom dimension
}

\author{
Goran Kolarević1,2*, Dražan Jaroš ${ }^{1,2}$, Bojan Pavičar ${ }^{1}$, Tatjana Ignjić1 , Aleksandar Kostovski1 , \\ Goran Marošević ${ }^{1,2}$, Branko Predojević3 ${ }^{3}$ Dragoljub Mirjanić2,3
}

${ }^{1}$ Center for Radiation Therapy, International Medical Centers, Affidea, Banja Luka, Bosnia and Herzegovina, ${ }^{2}$ Faculty of Medicine, University of Banja Luka, Bosnia and Herzegovina, ${ }^{3}$ Faculty of Natural Sciences and Mathematics, University of Banja Luka, Bosnia and Herzegovina

\begin{abstract}
Introduction: Using computed tomography (CT) and treatment planning systems (TPS) in radiotherapy, due to the difference in photon beam energy on CT and linear accelerator, it is necessary to convert Hounsfield units (HU) to relative electron density (RED) values. The aim of this dosimetric study was to determine whether there is a significant effect of potential in the CT tube, field of view size (FOV), and phantom dimensions on the CT conversion curve CT-RED. The second aim is whether there are significant differences between the CT-RED obtained by the Computerized Imaging Reference Systems (CIRS) Thorax 002LFC phantom and the "reference" curve in the TPS, obtained by the CIRS 062M pelvis phantom, at the same CT conditions.
\end{abstract}

Methods: Heterogeneous CIRS 062M and CIRS Thorax 002LFC phantoms were used, which anatomically and dimensionally represent the human pelvis, head, and thorax, with a set of known RED inserts. They were scanned on a CT LightSpeed GE simulator and obtained CT-RED.

Results: The high voltage in the CT tube had a significant effect on the HU $(t=10.72, p<0.001)$ for RED values $>1.1$, while FOV as a parameter did not show statistical significance for the $062 \mathrm{M}$ pelvis phantom. Comparing the slopes (062M pelvis and head) of the CT-RED for RED $\geq 1.1$, the obtained value is $t=1.404$ $(p=0.163)$. In the case of a $062 \mathrm{M}$ pelvis and a 002LFC phantom, we have seen a difference in RED values (for the same $\mathrm{HU}$ value) of $5 \%$ in the RED region $\geq 1.1$ (bone).

Conclusion: Patients should be imaged on a CT simulator only at the potential of the CT tube on which the conversion curve was recorded. The influence of the FOV and scanned phantom dimensions is not statistically significant on the appearance of the calibration curve (RED $\geq 1.1$ ).

Keywords: Computed tomography simulator; computed tomography-relative electron density; heterogeneous phantom; Hounsfield units; relative electron density

\footnotetext{
*Corresponding author: Goran Kolarević, Center for Radiation Therapy, International Medical Centers-Affidea, Dvanaest beba bb, 78000 Banja Luka, Bosnia and Herzegovina.

E-mail: goran.kolarevic@affidea.com
}

Submitted: 25 September 2020/Accepted: 26 November 2020

DOI: https://doi.org/10.17532/jhsci.2020.1085

UNIVERSITY OF SARAJEVO FACULTY OF HEALTH STUDIES

\section{INTRODUCTION}

Computer treatment planning system (TPS) for dose calculation in radiotherapy (RT), based on computed tomography (CT) and tissue heterogeneity, was first developed in the 1970s (1). The improvement of computer technology in the last 
two decades has enabled the emergence of modern TPS in external beam radiation therapy, which has, with the use of modern CT units, enabled more precise planning of RT treatments and the introduction of three-dimensional conformal RT. Therefore, we can adjust the isodose distribution in the patient to the irregularly shaped target volume with the maximum possible sparing of the surrounding healthy tissue and organs at risk.

The shape of the calculated isodose distribution in the patient depends on the calculation algorithm and the data from the CT and the medical linear accelerator (LINAC) entered into the TPS (2). On the other hand, CT imaging provides clinical and physical information about the patient: The size, shape, and position of the lesion and data on tissue inhomogeneity by assigning the value of the Hounsfield unit (HU) to each pixel, which is defined as:

$$
\mathrm{CT}_{\text {number }}(\mathrm{HU})=\left[\left(\mu_{\text {tissue }}-\mu_{\text {water }}\right) / \mu_{\text {water }}\right] .1000
$$

where $\mu_{\text {tissue }}$ is the linear attenuation coefficient of a particular tissue type and $\mu_{\text {water }}$ for water, while $\mathrm{CT}_{\text {number }}$ represents the normalized (to water) value of the linear attenuation coefficient. Photon beam attenuation coefficient of one element depends on the relative electron density (RED), atomic number $(Z)$, and quality (energy) of the photon beam obtained from the CT scanner (3).

There are three effects that attenuate a photon beam of energy up to $1 \mathrm{MeV}$. These are photoelectric absorption on the one hand and coherent-elastic (Rayleigh-with more strongly bound electrons and Thomson-on weakly bound electrons that can be considered as free) and incoherent-inelastic scattering (Compton) on the other. The total attenuation coefficient is $(4,5)$ :

$\mu_{\text {tissue }}=\rho_{\mathrm{e}}\left(\sigma_{\mathrm{e}}^{\text {photo }}+\sigma_{\mathrm{e}}^{\text {coher }}+\sigma_{\mathrm{e}}^{\text {incoher }}\right)$

where $\rho_{\mathrm{e}}$ is the RED (number of electrons per unit volume of the observed material) and $\sigma_{e}^{\text {photo }} \sim \mathrm{Z}^{5} / \mathrm{E} \gamma^{3.5}$, $\sigma_{\mathrm{e}}^{\text {coherThom }} \sim \mathrm{R}_{\mathrm{e}}^{2}\left(\mathrm{R}_{\mathrm{e}}\right.$-classical electron radius), $\sigma_{\mathrm{e}}^{\text {coherRay }}$ $\mathrm{Z} / \mathrm{E} \gamma^{2}$, and $\sigma_{e}^{\text {incoherCompt }}-\mathrm{Z} / \mathrm{E} \gamma$, corresponding efficient cross-sections (scattering) for photon-electron interaction removing photons from the beam (5). As commercial CT simulators generate photon beam energy (80-140) keV and LINACs (4-18) $\mathrm{MeV}$, it is obvious that the $\mathrm{HU}$ data from the CT scanner cannot be used to calculate the dose delivered by a
LINAC. This problem is overcome by the so-called CT calibration-conversion curves (CT-RED), that is, by converting the HU to known values of RED or material density (which do not depend on the photon beam energy spectrum). These measurements were the subject of several studies $(1,3,4,6)$ which used special heterogeneous phantoms for this purpose. For materials in the range between -1000 $\mathrm{HU}$ (air) and $0 \mathrm{HU}$ (water), the conversion curve is a linear straight line, while for bone simulating materials ( $>300 \mathrm{HU}$ ), the HU values are above this line and the values vary depending on the type of the CT unit. This brings us to the recommendation to determine the conversion curve for each CT simulator used for planning in RT (3).

Only one conversion curve can be entered in the TPS for a particular CT simulator. For the calculation algorithms in the TPS, such as Analytical Anisotropic Algorithm, it is necessary to convert the $\mathrm{HU}$ to RED, that is, to a physical density $\left(\mathrm{g} / \mathrm{cm}^{3}\right)$ for AcurosXB.

The aim of this dosimetric study is to investigate whether there is a significant difference between the CT-RED obtained at different: CT tube potentials, CT field of views (FOV), and diameters of heterogeneous phantoms Computerized Imaging Reference Systems ([CIRS] 062M pelvis and head). Moreover, whether there are significant differences between the CT-RED obtained by the CIRS Thorax 002LFC phantom and the "reference" curve in TPS, obtained by the CIRS $062 \mathrm{M}$ pelvis phantom, at the same CT conditions.

\section{METHODS}

Under the same, standardized, and methodological principles, this study investigated the influence of different CT-RED parameters. All-round testing was carried out at the same facility in a short period of time by engaging the same professional team, which generally implies repeatability and accuracy of the measurements.

\section{Phantoms}

Phantom CIRS 062M (CIRS Inc., Norfolk VA, USA) allows precise correlation of the HU with the RED of different tissues and is made of tissue-equivalent materials. It consists of two water 
equivalent disks (Figure 1) that together dimensionally simulate the human pelvis (horizontal diameter $33 \mathrm{~cm}$ ), while only the inner part represents the human head (diameter $18 \mathrm{~cm})$. It has a water plug plus two sets of 8 plugs $(3 \mathrm{~cm}$ in diameter) with different tissue types of known RED and mass densities (Table 1), which can be placed in 17 different positions (7).

The CIRS Thorax 002LFC (Figure 2) phantom is a heterogeneous phantom that simulates the average human thorax (30 $\mathrm{cm}$ in diameter). It is made of plastic water, lungs, and bone replacements, with five different cylindrical plugs of known RED (Table 2) and mass densities (water, bone, muscle, adipose, and lung equivalent tissue) that can be placed in 10 different positions (8).

\section{Scanning the phantom on a CT simulator}

Phantoms $062 \mathrm{M}$ pelvis and head were scanned on a wide bore $(80 \mathrm{~cm})$, sixteen slice CT simulator LightSpeed (GE Medical Systems, Boston MA,

TABLE 1. CIRS 062M phantom relative electron and mass density data

\begin{tabular}{lcc}
\hline CIRS 062M & $\begin{array}{c}\text { Physical density } \\
\left(\mathrm{g} / \mathrm{cm}^{3}\right)\end{array}$ & $\begin{array}{c}\text { RED (relative } \\
\left.\text { to } \mathrm{H}_{2} \mathrm{O}\right)\end{array}$ \\
\hline Lung (inhale) 04-053 & 0.205 & 0.2 \\
Lung (exhale) LH-053 & 0.507 & 0.486 \\
Adipose 11-046 & 0.96 & 0.949 \\
Breast 06-027 & 0.99 & 0.976 \\
Water 03-869 & 1.029 & 0.998 \\
Muscle 10-058 & 1.06 & 1.043 \\
Liver RDDOS-057 & 1.07 & 1.052 \\
Trabecular bone 08-038 & 1.16 & 1.117 \\
Dense bone 07-042 & 1.53 & 1.456 \\
\hline
\end{tabular}

RED: Relative electron density

TABLE 2. Thorax 002LFC phantom relative electron and mass density data

\begin{tabular}{lcc}
\hline Thorax 002LFC & $\begin{array}{c}\text { Physical density } \\
\left(\mathrm{g} / \mathrm{cm}^{3}\right)\end{array}$ & RED (relative to $\left.\mathrm{H}_{2} \mathrm{O}\right)$ \\
\hline Lung & 0.21 & 0.207 \\
Adipose & 0.96 & 0.949 \\
Water & 1 & 1 \\
Muscle & 1.06 & 1.042 \\
Bone core & 1.6 & 1.506 \\
\hline
\end{tabular}

RED: Relative electron density
USA), at CT tube potential of $80 \mathrm{kV}, 100 \mathrm{kV}$, $120 \mathrm{kV}$, and $140 \mathrm{kV}$ (helical-scan type, image slice thickness $1.25 \mathrm{~mm}$, current $400 \mathrm{~mA}$, tube rotation time $1 \mathrm{~s}, 512 \times 512$ matrix size, and standard WideView reconstruction algorithm) and FOVs of $25 \mathrm{~cm}$ and $50 \mathrm{~cm}$. The phantom Thorax 002LFC was scanned at only $120 \mathrm{kV}$ (FOV $50 \mathrm{~cm}, 400 \mathrm{~mA}$, $1.25 \mathrm{~mm}, 1 \mathrm{~s}$ ). Phantoms were scanned with cylindrical inserts of known RED (randomly positioned in phantoms) to obtain a CT-RED (HU = $\mathrm{f}$ $[R E D])$. The analysis of the obtained images where performed in the RT TPS Varian Eclipse 13.6 (Varian, Medical Systems, Palo Alto CA, USA). For each known material, the mean $\mathrm{HU}$ value and the standard deviation (SD) were read (based on 3 readings) as a measure of the variation of the $\mathrm{HU}$ value with respect to the CT scan parameters. To reduce the variations in the $\mathrm{HU}$ reading, a square region of interest was defined (side of $1 \mathrm{~cm}$ ), which excludes the edge zones of the inserts (Figure 3). The obtained

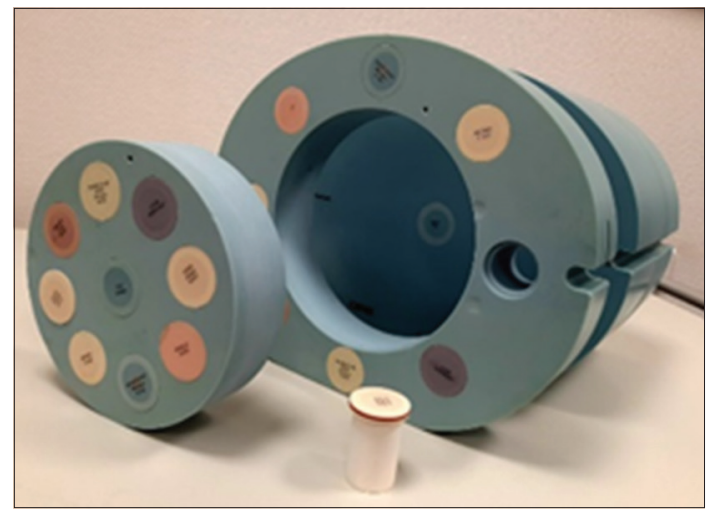

FIGURE 1. Computerized imaging reference systems 062M phantom.

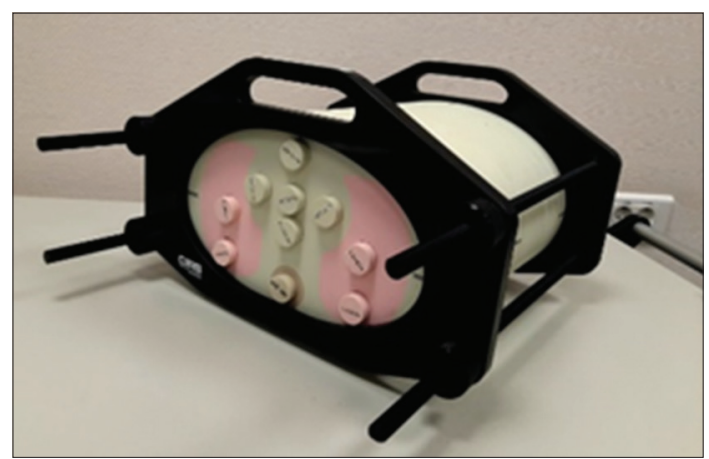

FIGURE 2. Thorax 002LFC phantom. 


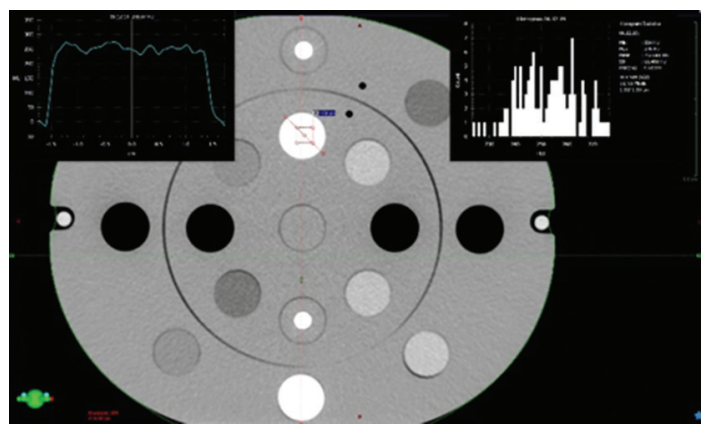

FIGURE 3. Axial Computed tomography scan of computerized imaging reference systems $062 \mathrm{M}$ pelvis phantom with region of interest analysis (Histogram and Hounsfield units profile).

conversion curves were divided and analyzed into two regions (2), RED $<1.1$ and $\mathrm{RED} \geq 1.1$.

In the TPS Eclipse 13.6, a "reference" conversion curve obtained based on data for the CIRS 002M pelvis phantom at $120 \mathrm{kV}$ (FOV $50 \mathrm{~cm}, 400 \mathrm{~mA}$, $1.25 \mathrm{~mm}, 1 \mathrm{~s}$ ) was entered. When comparing the conversion curves, the acceptable RED difference for the same $\mathrm{HU}$ value between them is \pm 0.02 (i.e., $\pm 20 \mathrm{HU}$ for the same RED value, except for water $\pm 5 \mathrm{HU}$ ) (9).

\section{Statistical analysis}

Results were presented as an arithmetic mean values with SD. Relationship and strength of the association between independent predictors (tissue types, tube potential, FOV, and phantom dimensions) and dependent variable (HU) were determined using multiple linear regression analyses (enter method).

To determine whether the slopes of two lines were significantly different from each other (linear regression analysis), the following formula was used:

$\mathrm{T}=\left(\mathrm{B}_{1}-\mathrm{B}_{2}\right) / \sqrt{ }\left(\mathrm{SE}_{1}{ }^{2}-\mathrm{SE}_{2}{ }^{2}\right)$

$\mathrm{B}_{1}$ and $\mathrm{B}_{2}$ are the slopes and $\mathrm{SE}_{1}$ and $\mathrm{SE}_{2}$ are the corresponding standard error values of regression lines (10). Complete statistical analysis of data was done with the statistical software package, SPSS Statistics 18 (IBM, Armonk NY, USA).

\section{RESULTS}

\section{CT-RED depending on tube potential and FOV}

By measuring the $\mathrm{HU}$ values (72 measuring points, 216 measurements) for known tissue types, that is, RED values, we obtained the data for CT-RED by the CIRS 062M pelvis phantom. The measurements on different CT tube potentials and FOVs are graphically presented in Figure 4.

Results of multiple linear regression analysis to the mean HU values of all measurement points (model based on all available data, $\mathrm{N}=216$ ) for the CIRS $062 \mathrm{M}$ pelvis phantom are presented in Table 3.

Declining (parallel) trend of the HU with increasing CT tube potential for trabecular and dense bone at different FOV values is presented in Figure 5.

The measured values of the HU do not depend on the CT tube potential and FOV for RED values $<1.1$, while for RED values $\geq 1.1$ (trabecular and dense bone), the difference depending on the tube potential is evident, and therefore, this area was further analyzed (Figure 4).

Using the multiple linear regression analysis, the effect of parameters (CT tube potential, FOV) on the $\mathrm{HU}$ was examined for $\mathrm{RED} \geq 1.1$ (Table 3 ).

\section{Conversion curve depending on the dimensions of the phantom}

By measuring the $\mathrm{HU}$ values (72 measuring points, 216 measurements) for known RED values, we obtained the data for CT-RED by the CIRS 062M-head phantom. We performed measurements on different CT tube potentials and FOV values, which are graphically presented in Figure 6.

The obtained results for the CIRS 062M head and pelvis phantoms were analyzed together. Figure 6 shows the CT-RED for pelvis and head phantoms at different CT tube potentials at FOV $25 \mathrm{~cm}$ and $50 \mathrm{~cm}$.

For RED values $<1.1$, the measured HU values do not depend on the phantom dimensions, while for RED values $\geq 1.1$ (trabecular and dense bone), a difference is observed (Figure 6). Thus, for $120 \mathrm{kV}$, FOV $50 \mathrm{~cm}$, the HU difference in the dense bone region is $9.7 \%$ (for the same value $\mathrm{RED}=1.456$ ), while for FOV $25 \mathrm{~cm}$, the difference is $11.6 \%$. Therefore, this part of the curve was additionally statistically analyzed.

Using the linear regression model, the slope of conversion curves for RED $\geq 1.1$ is presented (Table 4). Based on the formula (3) for slope and data comparison from Table 4, we obtain $t=1.404(p=0.163)$. 


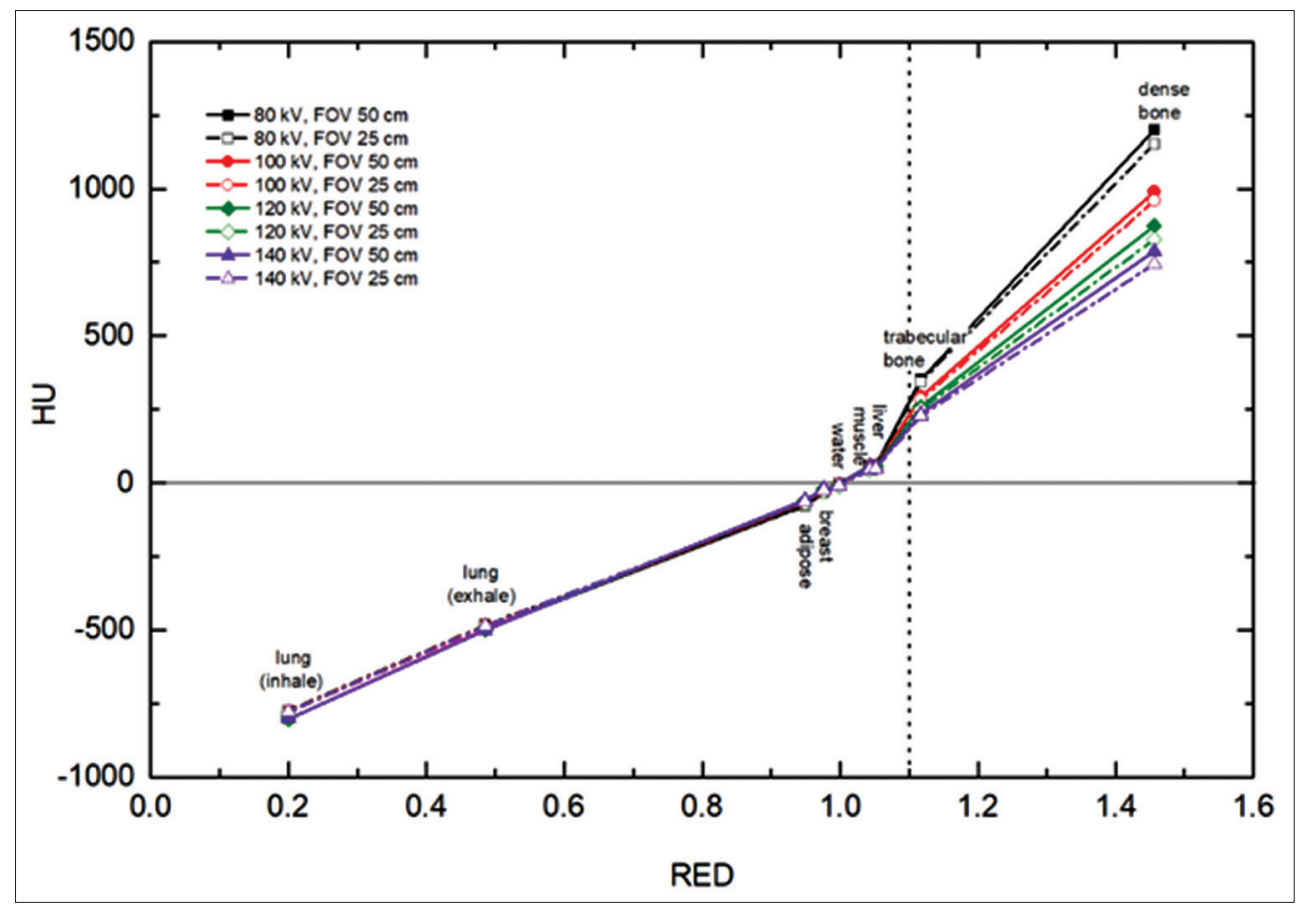

FIGURE 4. Computed tomography-relative electron density obtained by the computerized imaging reference systems $062 \mathrm{M}$ pelvis phantom, for different tube potentials ( $80 \mathrm{kV}, 100 \mathrm{kV}, 120 \mathrm{kV}$, and $140 \mathrm{kV}$ ) and field of view size $25 \mathrm{~cm}$ and $50 \mathrm{~cm}$.

TABLE 3. Multiple linear regression analysis (CIRS 062M-pelvis), effect of the CT tube potential and FOV on the mean HU values, for RED values $0.2-1.456$ and just for $\geq 1.1$

\begin{tabular}{|c|c|c|c|c|c|c|c|c|}
\hline \multicolumn{9}{|c|}{ Validity: Adjusted $R^{2}=0.816 ; F=318.83(p<0.001)$} \\
\hline \multirow[t]{2}{*}{ CIRS062M-pelvis } & \multirow[t]{2}{*}{ Model } & \multicolumn{2}{|c|}{$\begin{array}{l}\text { Unstandardized } \\
\text { Coefficients }\end{array}$} & \multirow{2}{*}{$\begin{array}{c}\text { Standardized } \\
\text { Coefficients } \\
\text { Beta }\end{array}$} & \multirow[t]{2}{*}{$t$} & \multirow[t]{2}{*}{$p$} & \multicolumn{2}{|c|}{$\begin{array}{l}95 \% \text { Confidence } \\
\text { Interval (B) }\end{array}$} \\
\hline & & B & SE & & & & Lower & Upper \\
\hline \multirow[t]{3}{*}{ RED (0.2-1.456) } & Tissue types (RED) & 159.43 & 5.16 & 0.904 & 30.88 & 0.000 & 149.25 & 169.60 \\
\hline & CT tube potential & -0.94 & 0.59 & -0.046 & 1.58 & 0.114 & -2.12 & 0.22 \\
\hline & FOV & 0.19 & 1.06 & 0.005 & 0.17 & 0.858 & -1.91 & 2.29 \\
\hline \multicolumn{9}{|c|}{ Validity: Adjusted $\mathrm{R}^{2}=0.969 ; \mathrm{F}=487.80(p<0.001)$} \\
\hline \multirow[t]{3}{*}{$\operatorname{RED}(\geq 1.1)$} & Tissue types (RED) & 661.44 & 18.02 & 0.94 & 36.69 & 0.000 & 661.44 & 18.02 \\
\hline & CT tube potential & -4.32 & 0.40 & -0.27 & 10.72 & 0.000 & -4.32 & 0.40 \\
\hline & FOV & 1.00 & 0.72 & 0.03 & 1.39 & 0.169 & 1.00 & 0.72 \\
\hline
\end{tabular}

Dependent Variable: HU

Independent predictors: Tissue types (RED), CT tube potential, FOV

$\mathrm{R}^{2}$-coefficient of multiple determination, CT: Computed tomography, FOV: Field of view size, HU: Hounsfield units, RED: Relative electron density

\section{Correlation of CT-REDs for 062M pelvis and Thotrax002LFC phantoms}

By measuring the HU values for known RED values, we obtained the CT-RED for the CIRS Thorax 002LFC phantom. The obtained curve was compared with the "reference" CIRS 062M pelvis CT-RED (Figure 7), at a CT tube potential of $120 \mathrm{kV}$ and FOV $50 \mathrm{~cm}$.

Where (Figure 7) the difference in the area of large RED (bones) is seen, while in the lower density region, the match is within the allowed values (9). The RED values for bones (649 HU) differ by 5 
$\%$, while the difference in the HU (RED 1.4) is $16 \%$.

Using the linear regression model, we compared the slope conversion curves for $062 \mathrm{M}$ pelvis and 002LFC phantom (Table 4).

Based on the formula (3) for comparing the two slopes and the data from Table 4, we obtain $t=1.334$ $(p=0.1895)$.

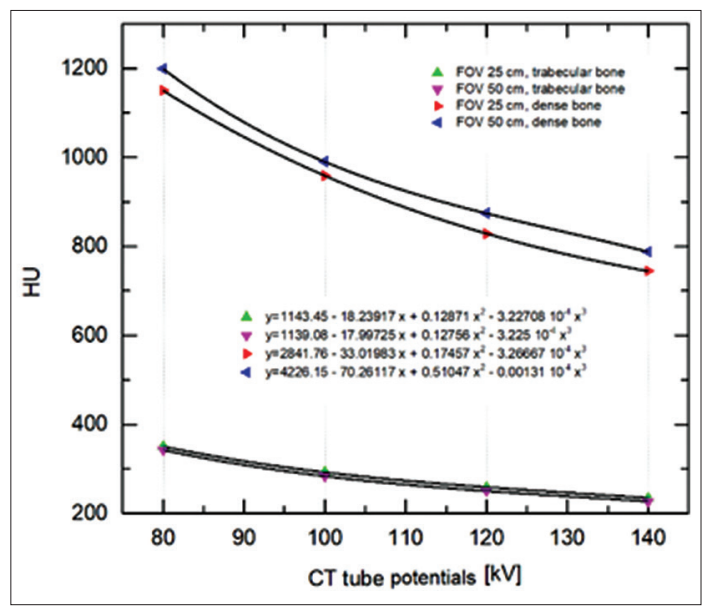

FIGURE 5. Decrease in Hounsfield units value with increasing tube potentials for trabecular and dense bone, for field of view size $25 \mathrm{~cm}$ and $50 \mathrm{~cm}$.

\section{DISCUSSION}

a. Based on the multiple regression analysis for all CIRS 062M pelvis phantom tissues, there is not a significant influence of the CT tube potential and FOV on the HU (Table 3).

It is obvious that the differences in the read HUs, depending on the CT tube potential, are evident only in tissues of higher density (trabecular and dense bone) and that the HU values decrease (with the increase of the CT tube potential in the bones area (Figure 4).

Given that the curves $\mathrm{HU}=\mathrm{f}$ (CT tube potential) are parallel (Figure 5) indicates that FOV, as a parameter, has no statistical significance.

Despite the significant decrease in the number of analyzed tissue types (processed only RED values $\geq 1.1$, Table 3), the adjusted coefficient of determination $\left(\mathrm{R}^{2}=0.969 \approx 97 \%\right)$ indicates that the three selected parameters explain the changes in the $\mathrm{HU}$ values as much as possible. Thanks to this, a high significance of the ANOVA test was achieved $(\mathrm{F}=487.89)$, all of which indicate good characteristics of the chosen linear regression model. In this case, not only tissue type but also the CT tube potential $(t=10.72, p<0.001)$ has a significant influence, which is in accordance with the results of the studies by Zurl et al. (2). The
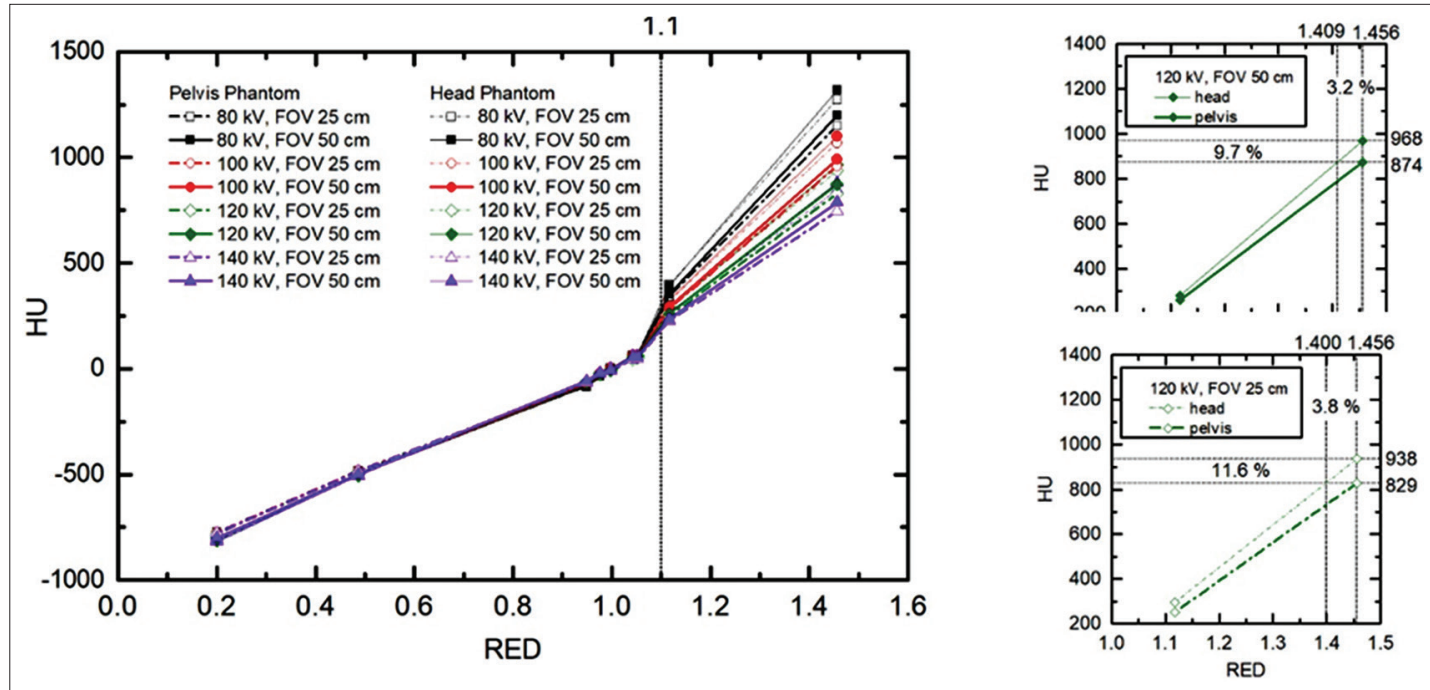

FIGURE 6. Change of the Hounsfield units depending on the relative electron density at different values of computed tomography tube potentials and field of view sizes for computerized imaging reference systems 062M pelvis and head phantoms. 
TABLE 4. Linear regression models for CIRS 062M-pelvis and head, based only on cases for RED $\geq 1.1$ and CIRS 062M-pelvis and Thorax 002LFC phantom based on cases for $1.506 \geq$ RED $\geq 0.2$

\begin{tabular}{|c|c|c|c|c|c|}
\hline \multirow[t]{2}{*}{ Model } & & \multicolumn{2}{|c|}{ Unstandardized Coefficients } & \multirow[t]{2}{*}{$t$} & \multirow[t]{2}{*}{$p$} \\
\hline & & B & SE & & \\
\hline \multirow[t]{3}{*}{ CIRS 062M-pelvis (RED $\geq 1.1$ ) } & $R=0.945$ & & & & \\
\hline & (Constant) & -1899.28 & 129.112 & -14.71 & 0.000 \\
\hline & RED & 1951.17 & 99.49 & 19.61 & 0.000 \\
\hline \multirow[t]{3}{*}{ CIRS 062M-head (RED $\geq 1.1$ ) } & $R=0.949$ & & & & \\
\hline & (Constant) & -2087.26 & 136.41 & -15.30 & 0.000 \\
\hline & RED & 2154.36 & 105.12 & 20.49 & 0.000 \\
\hline \multirow[t]{3}{*}{ CIRS 062M-pelvis $(1.506 \geq$ RED $\geq 0.2)$} & $R=0.986$ & & & & \\
\hline & (Constant) & -1046.839 & 45.024 & -23.251 & 0.000 \\
\hline & RED & 1128.784 & 47.981 & 23.557 & 0.000 \\
\hline \multirow[t]{3}{*}{ Thorax 002LFC (1.506 $\geq$ RED $\geq 0.2)$} & $R=0.971$ & & & & \\
\hline & (Constant) & -1149.55 & 60.081 & -19.133 & 0.000 \\
\hline & RED & 1232.439 & 61.151 & 20.154 & 0.000 \\
\hline
\end{tabular}

Dependent Variable: HU

Predictors: (Constant), RED

R: Coefficient of correlation, SE: Standard error, B: The slope of conversion curves, RED: Relative electron density

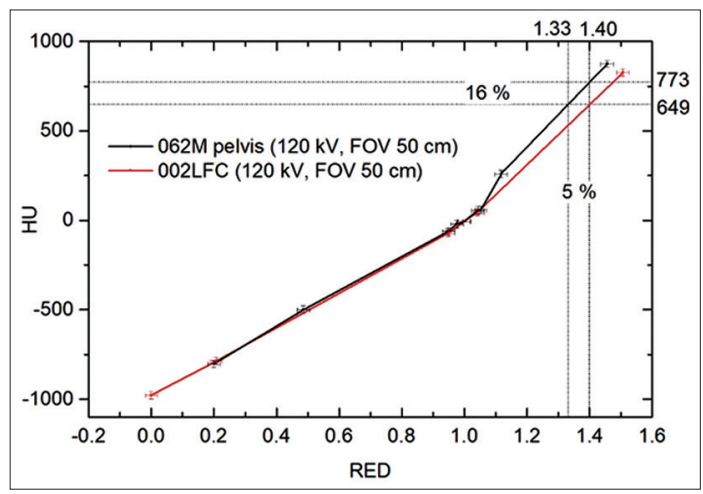

FIGURE 7. Computed tomography-relative electron density for Thorax 002LFC and computerized imaging reference systems $062 \mathrm{M}$ pelvis phantoms at $120 \mathrm{kV}$ and field of view size $50 \mathrm{~cm}$.

CT tube potential is a significant predictor only in the $\mathrm{RED} \geq 1.1$ region. In general, the deviations are the smallest with low-density materials and become larger with increasing density.

b. The HU differences in the two phantoms (pelvis and head) were found to increase as a function of RED (Figure 6). In the soft tissue region, the difference is $<10 \mathrm{HU}$ (for the same RED value), while for the dense bone material, the difference increases to around $100 \mathrm{HU}$, which is in accordance with the results of the studies of James et al. (11).
As Ebert et al. (12) have shown, there is a trend of an increase in the HU number with a decreasing phantom diameter, particularly for the high-density materials. Our results clearly show the same effect for the dense bone (Figure 6).

Using the linear regression model, we compared the slope conversion curves for RED $\geq 1.1$ and obtained high values of the correlation coefficients for both phantoms (pelvis $\mathrm{R}=0.945$ and head $\mathrm{R}=0.949$ ) but also high values of the standard error (Table 4). The obtained T value $1.404(p=0.163)$ is not significant, so these two sections-slopes for RED $\geq 1.1$ are comparable and we cannot say that the CT-RED depends on the dimensions of these two phantoms.

c. In the case of the $062 \mathrm{M}$ pelvis and 002LFC phantom, we see a difference in RED values (for the same $\mathrm{HU}$ value) of $5 \%$ in the RED $\geq$ 1.1 (bone) region by comparing the CT-RED (which is in accordance with the results of the studies Kolarević et al.) (13), while in the lower density region, the match is within the allowed values (9) (Figure 7).

However, it is estimated that a difference of $8 \%$ in the bone RED affects the TPS dose calculation accuracy by less than 1\% (3). Similarly, Geise et al. (14) reported that a (4-10)\% uncertainty in RED would result in no more than a $2 \%$ change in dose. 
The obtained value $t=1.334(p=0.1895)$ indicates that there is no statistically significant difference between the slopes of the CT curves for the 062M pelvis and 002LFC phantoms.

\section{CONCLUSION}

In clinical practice, patients on a CT simulator should be imaged only at the tube potential at which the conversion curve was recorded. Consider introductory possibilities for the external beam TPS for one CT simulator to have an option with multiple calibration curves for different tube potentials, FOVs, and diameter-dimensions.

The Thorax 002LFC phantom can be used to control the CT-RED (as part of the End-to-End QA test) but not to initially define it.

\section{REFERENCES}

1. Ahnesjo A, Aspradakis M. Dose calculations for external photon beams in radiotherapy. Phys Med Biol 1999;44(11):R99-155.

https://doi.org/10.1088/0031-9155/44/11/201.

2. Zurl B, Tiefling R, Winkler $P$, Kind $K$, Kapp KS. Hounsfield units variations-impact on CT density based conversion tables and their effects on dose distribution. Strahlenther Onkol 2014;190:88-93.

https://doi.org/10.1007/s00066-013-0464-5.

3. Thomas SJ. Relative electron density calibration of CT scanners for radiotherapy treatment planning. Br J Radiol 1999;72(860):781-6. https://doi.org/10.1259/bjr.72.860.10624344.

4. Trujillo C, Garcia O, Larraga J, Martinez A, Rodriguez M. Effective
Atomic Number and Electron Density Calibration with a Dual-Energy CT Technique, Medical Physics. AIP Conference Proceedings No. 1747; 2016. https://doi.org/10.1063/1.4954129.

5. Jackson DF, Hawkes DJ. X-Ray attenuation coefficients of elements and mixtures. Phys Rep 1981;70(3):169-233.

https://doi.org/10.1016/0370-1573(81)90014-4.

6. Cozzi L, Fogliata A, Buffa F, Bieri S. Dosimetric impact of computed tomography calibration on a commercial treatment planning system for external radiation therapy. Radiother Oncol 1998;48:335-8.

https://doi.org/10.1016/s0167-8140(98)00072-3.

7. Computerized Imaging Reference Systems. User Guide: Electron Density Phantom Model 062M. CIRS 2013:2.

8. Computerized Imaging Reference Systems. User Guide: IMRT Thorax Phantom Model 002LFC. CIRS 2013:2.

9. IAEA. Commissioning and Quality Assurance of Computerized Planning Systems for Radiation Treatment Planning Systems, TRS No.430. Vienna: IAEA; 2004.

https://doi.org/10.1118/1.2167371.

10. Spasic S, Kotur J, Bogavac N. Primenjena Statistika: Za Studente Farmacije. Beograd: Farmaceutski Fakultet; 2019.

11. Chua J, Nia B, Krizc R, Saxenab A. Applications of simulator computed tomography number for photon dose calculations during radiotherapy treatment planning. Radiother Oncol 2000;55(1):65-73.

https://doi.org/10.1016/s0167-8140(00)00159-6.

12. Ebert MA, Lambert J, Greer PB. CT-ED conversion on a GE lightspeed-RT scanner: Influence of scanner settings. Australas Phys Eng Sci Med 2008;31(2):154-9.

https://doi.org/10.1007/bf03178591.

13. Kolarević $G$, Jaroš $D$, Marošević $G$, Ignjatić $D$, Mirjanić $D$. Dosimetric verification of clinical radiotherapy treatment planning system. Vojnosanit Pregl 2020;1:70.

https://doi.org/10.2298/vsp200411070k.

14. Geise RA, McCullough EC. The use of CT scanners in megavoltage photon beam therapy planning. Radiology 1977;124(1):133-41.

https://doi.org/10.1148/124.1.133. 